\title{
Effectiveness of the Public Financial Management Assessment Tool or (PFMAT) in Strengthening the Financial Capability of Local Government unit in Talavera, Nueva Ecija
} \author{
Diaz $^{4}$, Sheela Mae C. Tuapen ${ }^{5}$, Marilou P. Pascual ${ }^{6}$ \\ ${ }^{1}$ Municipality of Talavera, Nueva Ecija, Philippines \\ ${ }^{2}$ Municipality of Rizal, Nueva Ecija, Philippines \\ ${ }^{3}$ RCS Eastern Intertrade Corp, Philippines \\ ${ }^{4}$ City Government of Cabanatuan, Nueva Ecija, Philippines \\ ${ }^{5}$ First Isabela Cooperative Bank, Philippines \\ ${ }^{6}$ Business Administration Department, NEUST Graduate School, Philippines
}

Rochelle b. Lapuz ${ }^{1}$, Aileen B. Macaculop ${ }^{2}$, Marc Lenon S. Del Rosario ${ }^{3}$, Jessy Alice M.

Received: 08 Apr 2021; Received in revised form: 20 May 2021; Accepted: 02 Jun 2021; Available online: 14 Jun 2021

\begin{abstract}
In both theoretical and practical areas, the issue of public sector management and the use of public finances is highly critical. The management of public finances is vital if public services, like local government, are to operate smoothly and effectively. If public financial management is such a vital element in a country's growth, it is crucial to know, quantify and develop the factors leading to poor public financial management. This descriptive research interest is on the effectiveness of the public financial management assessment tool or PFMAT for local government units. The chosen subject of the study is the Municipality of Talavera, Nueva Ecija, and the focus is on the results of the PFMAT report of the municipality for the covered fiscal years 2014-2016 and 2016-2018. The study revealed that the PFMAT is a standard tool that states the weak areas of the public financial performance of LGU-Talavera, thus paving the way for improved services for the residents of the municipality. LGU-Talavera has gradually improved its public financial performance in 2014-2018. PFMAT is therefore an effective tool for evaluating public finances to strengthen the town's financial capability, thereby enhancing the overall performance of the $L G U$.
\end{abstract}

Keywords-Assessment tool, effectiveness, financial capability, local government unit, public financial management system.

\section{INTRODUCTION}

For several years, research has centered on the fair utilization and efficient control of public finances. Evermore important for the smooth and productive operation of public institutions, like local government, in the sense of social and economic transition [1].Borrowing, raising, and spending of public finances are guided by a key tool, Public Financial Management (PFM) systems, which translate policy statements into the delivery of
services[2].PFM provides a general framework for revenue generation, spending control, and resource allocation which are essentially linked to the execution of LGU mandates[3].If there is strong financial control, and openness in the use of public funding, the public will have more trust in public sectors [4].Governments need to make the right decision as this impacts the areas in fiscal sustainability, operational management, governance, and fiduciary risk management [5].Several PFM diagnostics were introduced in the 1990s, the International Monetary 
Fund Code of Good Practices for Fiscal Transparency (1998), Standard and Codes Observance Report (ROSC), Public Expenditure and Financial Accountability (PEFA,2002), and International Budget Partnership's Open Budget Survey [6]. A comprehensive review of fiscal transparency and of how the PFM systems guarantee fiscal discipline, promote good financial management, and safeguard against fiscal risk every two years in 100 countries by civil society groups. The tool has four components included in each pillar, differentiating between basic, good, and advanced practices[7].Budget Practices and Procedures queries from 26 countries in Africa compare budget practice structures, including budget plans, off-budget expenditure, aid management, and liquidity[8].The 2013 amended PEFA Program aims to assess public spending, financial accountability, and procurement process. The World Bank, the IMF, and the European Commission all developed it[9].The framework provides the basis for evidence-based measurement of PFM systems by a set of indicators at the national level [10].International standards are used with the PEFA Methodology. A country-led approach to enhancing efficiency and getting stakeholders into line with shared priorities[11].PEFA reports outline the economic environment faced by the public sector, examine the nature of policy-based strategy and planning, and analyze how budget decisions are implemented[12].An uncoordinated evolution of a wide array of assessment tools noted that diagnostics did not give users an objective way of measuring progress in addressing PFM system weaknesses thus creates gaps and twofold coverage. Country ownership of diagnostics was lacking, reflected in a variety of action plans externally driven [13].

On the local level, fiscal controls will be strengthened by an automated system to plan the accrual, operational, and reporting budgets to satisfy multiple stakeholders' needs. The cash basis does not measure the money spent over the review period, so real government services and project expenses are misleading. Without reliable cost statistics, productivity and cost-effectiveness steps cannot easily be calculated[14].In the correlational study of six localities in Tanzania, councils with better financial management practices, budget planning, and project implementation achieve better results in the local management of financial resources[15].

The history of the Philippines' post-war squandering progress by poor economic governance, civic transparency, and the need for a holistic PFM overhaul has become increasingly evident in the new century [16].The route map concentrated primarily on a new Government Integrated Financial and Management Information System (GIFMIS) and PFM competency system and improvement of internal control audit pursuit the conclusion of the first PEFA report in 2006 [17]. The key was to create an integrated financial information framework, adoption of the performance-based budget, procurement planning, individual treasury account, CSO management engagement, and establishment of substantive PFM legislation [18].The LGUs Public Financial Management Assessment Tool was created by a team of experts in 2010 . The initial version was based on the PEFA launched framework and made terminologies that suited the Philippine locale[19].It helps LGUs to calculate their PFM results. Outlines an effective PFM system, establishes efficiency metrics to analyze how the LGU works in a certain region of PFM [20]PFM is strictly mediated by the Local Government Code for LGU on related taxation and fiscal matters. [21] The growing demand for systematic and consistent monitoring of the LGUs - PFM system makes the researchers interested in how the Philippines PFM Assessment improves the financial performance of the LGU. It is specifically intended to:Discuss the condition of public financial management in the LGUTalavera from covering fiscal years 2014-2016 and 20162018 established on the pursuit of critical dimensions;(a) Policy-based budgeting, (b) Comprehensive and Transparency, (c) Budget Credibility, (d) Predictability and control in the budget execution, (e) Accounting, recording, and reporting, (f) Internal and external audit, and (g) Participation of Citizens. Also, this revealed the best practices and pitfalls of LGU Talavera in implementing its Public Financial Management Systems.

\section{METHODOLOGY}

The researchers used the descriptive research design. Gay (1992) states that it gathers data to address inquiries and test theories on the current subject [22]. The sample study was made in the Municipality of Talavera, a first-class town in Nueva Ecija. propelled by its governance thrust: Nagbibigay ng Serbisyo at Malasakit. It is one of the progressive municipalities in Nueva Ecija and proposals have been planned for cityhood [23]. With this progress, it is important to measure the financial capability and examine if there are performance improvements or failures brought about, partly, or whole in the municipality based on the results of PFMAT. A self-assessment instrument for LGUs is the Public Financial Management Assessment Tool used as a research instrument. The data presentation is constructed using the orderly and open PFM systems' seven critical dimensions twenty (20) performance indicators and interpreted using the provided Interpretation Guide by the Department of Budget and Management on the PFMAT Book p.19 [24]. Every LGU has a PFM team assigned to respond to each achievement to indicators and 
sub-indicators. [25] Links to the PFMAT summarized results and online submission is given, and the researcher gathered the available data to the LGU-Talavera Municipal Budget Officer and was followed up by the respective offices responsible for the analysis. The statistical treatment used is a percentage and averaging. If the previous year's weak areas changed during previous rating cycles, the tool has been able to assess and monitor LGU performance effectively. Improved LGU performance enhances basic amenities and improves people's quality of life.

The study used the logic model (also known as a logical framework, or program matrix), a tool created by
Martin Quigley to evaluate the effectiveness of a program [26]. The frameworkused the result of PFMAT fiscal years 2014-2018, was not only concerned with measurement such as collecting data, analyzing variances, and reporting results but in managing LGU performance by developing PFM action plans to close those gapsto find out if PFMAT is an effective tool to strengthen financial capability of LGU-Talavera [27].

\section{RESULTS AND DISCUSSION}

The following are the summarized results of the PFMAT rating of the LGU-Talavera, data for each critical dimension and performance indicator are presented below.

Table 1. Public Financial Management (PFM) Assessment Result for the Local Government Unit of Talavera for the period 2014-2016 \& 2016-2018

\begin{tabular}{|c|c|c|c|c|}
\hline \multirow{2}{*}{$\begin{array}{l}\text { Indicator } \\
\quad \text { No. }\end{array}$} & \multicolumn{2}{|c|}{ SUMMARY OF LGU SCORES, BY CRITICAL DIMENSION } & \multicolumn{2}{|c|}{ SCORE } \\
\hline & CRITICAL DIMENSION & INDICATOR & $\begin{array}{l}2014- \\
2016\end{array}$ & $\begin{array}{l}2016- \\
2018\end{array}$ \\
\hline 1 & \multirow{4}{*}{$\begin{array}{l}\text { 1. POLICY-BASED } \\
\text { BUDGETING }\end{array}$} & Multi-year perspective in fiscal planning and budgeting & 3.67 & 4.00 \\
\hline 2 & & $\begin{array}{l}\text { PFM improvement policies are included in the budgets } \\
\text { covered by appropriation ordinances }\end{array}$ & 3.00 & 3.00 \\
\hline 3 & & $\begin{array}{l}\text { The orderliness of activities in the annual budget preparation } \\
\text { and authorization phases }\end{array}$ & 3.33 & 3.67 \\
\hline 4 & & $\begin{array}{l}\text { Financial self-reliance of local economic enterprises (LEEs) } \\
\text { and public utilities (PUs) }\end{array}$ & 4.00 & 4.00 \\
\hline \multicolumn{3}{|c|}{ Mean Score for policy-based budgeting } & 3.50 & 3.67 \\
\hline 5 & \multirow{2}{*}{$\begin{array}{l}2 . \\
\text { COMPREHENSIVENESS } \\
\text { AND TRANSPARENCY }\end{array}$} & $\begin{array}{l}\text { The comprehensiveness of budget information contained in } \\
\text { the appropriation ordinance covering the annual budget }\end{array}$ & 4.00 & 4.00 \\
\hline 6 & & Public access to key information & 3.00 & 3.00 \\
\hline \multicolumn{3}{|c|}{ Mean Score for Comprehensiveness and Transparency } & 3.50 & 3.50 \\
\hline 7 & \multirow{2}{*}{$\begin{array}{l}\text { 3. CREDIBILITY OF THE } \\
\text { BUDGET }\end{array}$} & $\begin{array}{l}\text { Actual revenue collections compared with estimated revenues } \\
\text { in the budget }\end{array}$ & 3.00 & 4.00 \\
\hline 8 & & $\begin{array}{l}\text { Actual expenditures compared with appropriations, by } \\
\text { allotment class }\end{array}$ & 3.67 & 3.67 \\
\hline \multicolumn{3}{|c|}{ Mean Score for the credibility of the budget } & 3.33 & 3.83 \\
\hline 9 & \multirow{6}{*}{$\begin{array}{l}\text { 4. PREDICTABILITY } \\
\text { AND CONTROL IN } \\
\text { BUDGET EXECUTION }\end{array}$} & Real property tax accomplishment rate (RPTAR) & 0.00 & 0.00 \\
\hline 10 & & Effectiveness of tax enhancement measures & 3.00 & 1.67 \\
\hline 11 & & $\begin{array}{l}\text { Predictability in the availability of cash for committed } \\
\text { expenditures }\end{array}$ & 4.00 & 4.00 \\
\hline 12 & & Value for money and controls in procurement & 2.60 & 1.60 \\
\hline 13 & & Effectiveness of payment controls & 4.00 & 4.00 \\
\hline 14 & & $\begin{array}{l}\text { Effectiveness of internal controls for non-personal services } \\
\text { (PS) expenditures }\end{array}$ & 4.00 & 4.00 \\
\hline \multicolumn{3}{|c|}{ Mean Score for predictability and control in budget execution } & 2.93 & 2.54 \\
\hline
\end{tabular}




\begin{tabular}{|c|c|c|c|c|}
\hline 15 & \multirow{2}{*}{$\begin{array}{l}\text { 5. ACCOUNTING, } \\
\text { RECORDING, AND } \\
\text { REPORTING }\end{array}$} & Timeliness and regularity of accounts reconciliation & 4.00 & 4.00 \\
\hline 16 & & $\begin{array}{l}\text { Quality and timeliness of regular financial reports and annual } \\
\text { financial statements }\end{array}$ & 4.00 & 4.00 \\
\hline \multicolumn{3}{|c|}{ Mean Score for the credibility of the budget } & 4.00 & 4.00 \\
\hline 17 & \multirow{2}{*}{$\begin{array}{l}\text { 6. INTERNAL AND } \\
\text { EXTERNAL AUDIT }\end{array}$} & Effectiveness of internal audit & 0.67 & 3.67 \\
\hline 18 & & Follow-up on external audit & 2.67 & 2.50 \\
\hline \multicolumn{3}{|c|}{ Mean Score for internal and external audit } & 1.67 & 3.08 \\
\hline 19 & \multirow{2}{*}{$\begin{array}{l}\text { 7. CITIZEN'S } \\
\text { PARTICIPATION }\end{array}$} & $\begin{array}{l}\text { Civil society organization (CSO) accreditation by local } \\
\text { sanggunian }\end{array}$ & 4.00 & 4.00 \\
\hline 20 & & Degree of citizen's participation in the budget process & 3.00 & 4.00 \\
\hline \multicolumn{3}{|c|}{ Mean Score for citizen's participation } & 3.50 & 4.00 \\
\hline \multicolumn{3}{|c|}{ OVERALL PFMAT SCORE } & 3.20 & 3.52 \\
\hline
\end{tabular}

Table 1 displays the summarized result of the PFMAT for the LGU-Talavera from the years 2014-2016 \& 20162018. It shows that from the covering fiscal years, the overall PFMAT score improved by 0.32 . Then, the highlighted mean score distribution of ratings by critical dimension reveals that Accounting, Recording, and Reporting consistently receive the highest score for covering fiscal years while Internal and External Audit dramatically increases from 1.67 to 3.08. On the other hand, Predictability and Control in Budget Execution decrease from 2.93 to 2.54.Furthermore, the distribution of ratings for fiscal years by indicators appears that indicators $5,11,13,14,15,16$, and 19 maintain the highest score while indicator 17 significantly increases contrary to decline performance for indicators 10,12 , and 18 as well the indicator 9 which remains the lowest. It is an indication in the assessment provided by PFMAT for LGUs that elements of an open and orderly PFM system of LGU Talavera are complete, nevertheless not fully operational.

Table 2. Indicator \#4 Financial self-reliance of Local Economic Enterprises (LEEs) and Public Utilities (PUs)

\begin{tabular}{|c|c|c|c|c|c|}
\hline Year & $\begin{array}{c}\text { Total Financial } \\
\text { Req. for } \\
\text { Operations of } \\
\text { All LEEs / Pus } \\
\text { (a) }\end{array}$ & $\begin{array}{c}\text { Total Income } \\
\text { of All LEEs/ } \\
\text { PUs }\end{array}$ & $\begin{array}{c}\text { Total LGU } \\
\text { Transfers to } \\
\text { LEEs / } \\
\text { PUs(c) }\end{array}$ & $\begin{array}{c}\text { \% of Total Financial } \\
\text { Requirements of all LEEs } \\
\text { Covered by Income of All } \\
\text { LEEs/ PUs (d=b/a) }\end{array}$ & $\begin{array}{c}\text { \% of Total Financial } \\
\text { Requirements of all LEEs } \\
\text { Covered by Transfers/ } \\
\text { Advances to LEEs/ PUs } \\
\text { (e=c/a) }\end{array}$ \\
\hline $\mathbf{2 0 1 8}$ & $12,976,905.00$ & $13,195,774.65$ & - & $\mathbf{1 0 2 \%}$ & $\mathbf{0 \%}$ \\
\hline $\mathbf{2 0 1 7}$ & $11,985,000.00$ & $12,846,241.78$ & - & $\mathbf{1 0 7 \%} \%$ \\
\hline $\mathbf{2 0 1 6}$ & $9,348,221.94$ & $13,186,804.70$ & - & $\mathbf{1 4 1} \%$ & $\mathbf{0} \%$ \\
\hline $\mathbf{2 0 1 5}$ & $8,638,190.47$ & $12,500,602.65$ & - & $\mathbf{1 4 5} \%$ & $\mathbf{0} \%$ \\
\hline $\mathbf{2 0 1 4}$ & $8,185,289.84$ & $14,381,292.31$ & - & $\mathbf{1 7 6} \%$ & $\mathbf{0} \%$ \\
\hline
\end{tabular}

Source/s: Last five years' Local Economic Enterprises and Public Utilities Financial Requirements for Operations

Table 2 shows that for all covering fiscal years, 100\% of the total financial requirements for operations of all LEEs / PUs were funded by their respective incomes. It is a special account funding charged against $20 \%$ of the IRA for development projects of LGUs, more than $100 \%$ signified that it is an income-generating project. 
Table 3. Indicator \#7. Actual Local Revenue Collections compared to Estimated Revenues in Budget

\begin{tabular}{|c|c|c|c|c|c|c|c|}
\hline \multirow[b]{2}{*}{ Year } & \multicolumn{7}{|c|}{ Income Type } \\
\hline & RPTs & $\begin{array}{c}\text { Income from } \\
\text { Business }\end{array}$ & $\begin{array}{c}\text { Other Local } \\
\text { Taxes }\end{array}$ & $\begin{array}{c}\text { Income from } \\
\text { LEE's/PUs }\end{array}$ & $\begin{array}{l}\text { Fees and } \\
\text { Charges }\end{array}$ & $\begin{array}{c}\text { Other } \\
\text { Income }\end{array}$ & TOTAL \\
\hline $\begin{array}{l}\mathbf{2 0 1 8} \\
\text { Estimated }\end{array}$ & $22,324,000.00$ & $16,900,000.00$ & $2,000,000.00$ & $12,976,905.00$ & $14,376,982.00$ & $25,000.00$ & $68,602,887.00$ \\
\hline Actual & $15,467,480.15$ & $23,445,663.70$ & $2,208,973.43$ & $13,195,774.65$ & $19,192,911.80$ & $94,304.93$ & $73,605,108.66$ \\
\hline$\%$ & $69 \%$ & $139 \%$ & $110 \%$ & $102 \%$ & $133 \%$ & $377 \%$ & $107 \%$ \\
\hline $\begin{array}{l}2017 \\
\text { Estimated }\end{array}$ & $19,310,000.00$ & $14,595,000.00$ & $2,000,000.00$ & $11,985,000.00$ & $12,085,000.00$ & $25,000.00$ & $60,000,000.00$ \\
\hline Actual & $11,345,072.40$ & $16,500,511.56$ & $2,044,521.23$ & $12,846,241.78$ & $16,428,332.14$ & $156,921.63$ & $59,321,600.74$ \\
\hline$\%$ & $59 \%$ & $113 \%$ & $102 \%$ & $107 \%$ & $136 \%$ & $628 \%$ & $99 \%$ \\
\hline $\begin{array}{l}\mathbf{2 0 1 6} \\
\text { Estimated }\end{array}$ & $17,103,300.00$ & $13,095,000.00$ & $2,050,000.00$ & $11,020,000.00$ & $9,587,100.00$ & $600,000.00$ & $53,455,400.00$ \\
\hline Actual & $10,085,956.75$ & $14,712,317.33$ & $1,685,936.17$ & $13,186,804.70$ & $14,302,443.52$ & $309,329.70$ & $54,282,788.17$ \\
\hline$\%$ & $59 \%$ & $112 \%$ & $82 \%$ & $120 \%$ & $149 \%$ & $52 \%$ & $102 \%$ \\
\hline $\begin{array}{l}\mathbf{2 0 1 5} \\
\text { Estimated }\end{array}$ & $14,985,000.00$ & $13,149,704.00$ & $2,050,000.00$ & $11,595,631.00$ & $12,081,304.00$ & $985,532.00$ & $54,847,171.00$ \\
\hline Actual & $9,056,907.60$ & $13,122,271.63$ & $1,556,852.30$ & $12,500,602.65$ & $12,297,328.28$ & $492,795.01$ & $49,026,757.47$ \\
\hline$\%$ & $60 \%$ & $100 \%$ & $76 \%$ & $108 \%$ & $102 \%$ & $50 \%$ & $89 \%$ \\
\hline $\begin{array}{l}\mathbf{2 0 1 4} \\
\text { Estimated }\end{array}$ & $12,951,317.00$ & $9,878,577.00$ & $2,000,000.00$ & $13,857,373.00$ & $10,069,813.00$ & $800,000.00$ & 49,557,080.00 \\
\hline Actual & $7,908,751.00$ & $9,899,007.82$ & $1,555,116.64$ & $14,381,292.31$ & $10,031,517.43$ & $211,910.05$ & 43,987,595.25 \\
\hline$\%$ & $61 \%$ & $100 \%$ & $78 \%$ & $104 \%$ & $100 \%$ & $26 \%$ & $89 \%$ \\
\hline
\end{tabular}

Source/s: Annual Budgets and Statement of Receipts \& Expenditures (SREs). EST. thru historical data of mandated organization and reasonable assessments/expected revenues for the ensuing fiscal year.

Table 3 presents that in the years 2016 to 2018, total actual local revenue collections were $90 \%$ to more than $100 \%$ of the estimated local revenues, while the year 2014 to 2015 were both $89 \%$. In this context, LGU realized the estimated revenues which contribute to the collection efficiency of the LGU. However, Real Property taxes still represent the largest source of untapped municipal revenue. Making real property taxes as the productive sources of municipal revenues should be the main priority of LGUs to further stimulate the accomplishment of the Annual Investment Program (AIP) of the LGU set for community development.

Table 4. Indicator \# 8 - Actual Expenditures Compared with Appropriations by Allotment Class

\begin{tabular}{|c|c|c|c|c|c|c|c|c|c|}
\hline \multicolumn{2}{|c|}{ Particulars } & \multicolumn{2}{|c|}{$\begin{array}{l}\text { Personal Services } \\
\text { (PS) }\end{array}$} & \multicolumn{2}{|c|}{$\begin{array}{c}\text { Maintenance and Other } \\
\text { Operating Expenses } \\
\text { (MOOE) }\end{array}$} & \multicolumn{2}{|c|}{ Capital Outlays } & \multicolumn{2}{|c|}{ TOTAL } \\
\hline $\begin{array}{c}\text { Allotments } \\
\text { as } \% \text { of }\end{array}$ & $\begin{array}{l}2018 \\
\text { (b/a) }\end{array}$ & $\begin{array}{c}107,636,771 \\
.85 /\end{array}$ & $\begin{array}{c}99.76 \\
\%\end{array}$ & $\begin{array}{c}168,478,945 \\
.92 /\end{array}$ & $99.85 \%$ & $\begin{array}{c}291,945,888 \\
.16 /\end{array}$ & $\begin{array}{c}99.98 \\
\%\end{array}$ & $\begin{array}{c}568,061,605 \\
.93 /\end{array}$ & $\begin{array}{c}100 \\
\%\end{array}$ \\
\hline
\end{tabular}




\begin{tabular}{|c|c|c|c|c|c|c|c|c|c|}
\hline \multirow[t]{5}{*}{$\begin{array}{l}\text { Appropriat } \\
\text { ions }\end{array}$} & & $\begin{array}{c}107,895,375 \\
.43\end{array}$ & & $\begin{array}{c}168,731,945 \\
.92\end{array}$ & & $\begin{array}{c}291,999,188 \\
.40\end{array}$ & & $\begin{array}{c}568,626,509 \\
.75\end{array}$ & \\
\hline & $\begin{array}{r}2017 \\
(\mathbf{f} / \mathrm{e})\end{array}$ & $\begin{array}{c}94,713,915 . \\
65 / \\
95,453,442 . \\
82\end{array}$ & $\begin{array}{c}99.23 \\
\%\end{array}$ & $\begin{array}{c}156,195,276 \\
.44 / \\
156,234,616 \\
.44\end{array}$ & $99.97 \%$ & $\begin{array}{c}55,601,423 . \\
18 / \\
55,601,423 . \\
18\end{array}$ & $100 \%$ & $\begin{array}{c}306,510,615 \\
.27 / \\
307,289,482 \\
.44\end{array}$ & $\begin{array}{c}100 \\
\%\end{array}$ \\
\hline & $\begin{array}{r}2016 \\
(\mathrm{j} / \mathrm{i})\end{array}$ & $\begin{array}{c}93,497,555 . \\
71 / \\
93,497,555 . \\
71\end{array}$ & $100 \%$ & $\begin{array}{c}134,286,234 \\
.13 / \\
134,286,234 \\
.13\end{array}$ & $100 \%$ & $\begin{array}{c}35,528,034 . \\
20 / \\
35,528,034 . \\
20\end{array}$ & $100 \%$ & $\begin{array}{c}263,311,824 \\
.04 / \\
263,311,824 \\
.04\end{array}$ & $\begin{array}{c}100 \\
\%\end{array}$ \\
\hline & $\begin{array}{l}2015 \\
(n / m)\end{array}$ & $\begin{array}{c}79,384,263 . \\
77 / \\
80,065,908 . \\
46\end{array}$ & $\begin{array}{c}99.15 \\
\%\end{array}$ & $\begin{array}{c}131,325,819 \\
.78 / \\
141,415,417 \\
.26\end{array}$ & $92.87 \%$ & $\begin{array}{c}133,599,623 \\
.28 / \\
143,099,623 \\
.28\end{array}$ & $\begin{array}{c}93.36 \\
\%\end{array}$ & $\begin{array}{c}344,309,706 \\
.83 / \\
364,580,949 \\
.00\end{array}$ & $94 \%$ \\
\hline & $\begin{array}{l}2014 \\
(\mathbf{r} / \mathbf{q})\end{array}$ & $\begin{array}{c}76,382,647 . \\
90 / \\
76,382,647 . \\
90\end{array}$ & $100 \%$ & $\begin{array}{c}101,616,327 \\
.36 / \\
104,086,917 \\
.75\end{array}$ & $97.63 \%$ & $\begin{array}{c}150,568,695 \\
.15 / \\
158,068,695 \\
.15\end{array}$ & $\begin{array}{c}95.26 \\
\%\end{array}$ & $\begin{array}{c}328,567,670 \\
.41 / \\
338,538,260 \\
.80\end{array}$ & $97 \%$ \\
\hline \multirow{5}{*}{$\begin{array}{c}\text { Obligation } \\
\mathrm{s} \text { as \% of } \\
\text { Allotments }\end{array}$} & $\begin{array}{l}2018 \\
(c / b)\end{array}$ & $\begin{array}{c}102,426,274 \\
.18 / \\
107,636,771 \\
.85\end{array}$ & $\begin{array}{c}95.16 \\
\%\end{array}$ & $\begin{array}{c}150,923,865 \\
.78 / \\
168,478,945 \\
.92\end{array}$ & $89.58 \%$ & $\begin{array}{c}75,239,569 . \\
49 / \\
291,945,888 \\
.16\end{array}$ & $\begin{array}{c}25.77 \\
\%\end{array}$ & $\begin{array}{c}328,589,709 \\
.45 / \\
568,061,605 \\
.93\end{array}$ & $58 \%$ \\
\hline & $\begin{array}{l}2017 \\
(\mathrm{~g} / \mathrm{f})\end{array}$ & $\begin{array}{c}91,847,254 . \\
36 / \\
94,713,915 . \\
65\end{array}$ & $\begin{array}{c}96.97 \\
\%\end{array}$ & $\begin{array}{c}140,580,063 \\
.89 / \\
156,195,276 \\
.44\end{array}$ & $90.00 \%$ & $\begin{array}{c}46,114,144 . \\
62 / \\
55,601,423 . \\
18\end{array}$ & $\begin{array}{c}82.94 \\
\%\end{array}$ & $\begin{array}{c}278,541,462 \\
.87 / \\
306,510,615 \\
.27\end{array}$ & $91 \%$ \\
\hline & $\begin{array}{l}2016 \\
(\mathbf{k} / \mathbf{j})\end{array}$ & $\begin{array}{c}88,261,444 . \\
19 / \\
93,497,555 . \\
71\end{array}$ & $\begin{array}{c}94.40 \\
\%\end{array}$ & $\begin{array}{c}117,405,433 \\
.51 / \\
134,286,234 \\
.13\end{array}$ & $87.43 \%$ & $\begin{array}{c}33,017,020 . \\
33 / \\
35,528,034 . \\
20\end{array}$ & $\begin{array}{c}92.93 \\
\%\end{array}$ & $\begin{array}{c}238,683,898 \\
.03 / \\
263,311,824 \\
.04\end{array}$ & $91 \%$ \\
\hline & $\begin{array}{l}2015 \\
(\text { o/n })\end{array}$ & $\begin{array}{c}77,735,071 . \\
35 / \\
79,384,263 . \\
77\end{array}$ & $\begin{array}{c}97.92 \\
\%\end{array}$ & $\begin{array}{c}114,098,017 \\
.93 / \\
131,325,819 \\
.78\end{array}$ & $86.88 \%$ & $\begin{array}{c}127,373,232 \\
.30 / \\
133,599,623 \\
.28\end{array}$ & $\begin{array}{c}95.34 \\
\%\end{array}$ & $\begin{array}{c}319,206,321 \\
.58 / \\
344,309,706 \\
.83\end{array}$ & $93 \%$ \\
\hline & $\begin{array}{l}2014 \\
(\mathrm{~s} / \mathrm{r})\end{array}$ & $\begin{array}{c}73,710,506 . \\
56 / \\
76,382,647 . \\
90\end{array}$ & $\begin{array}{c}96.50 \\
\%\end{array}$ & $\begin{array}{c}88,437,431 . \\
43 / \\
101,616,327 \\
.36\end{array}$ & $87.03 \%$ & $\begin{array}{c}137,625,942 \\
.21 / \\
150,568,695 \\
.15\end{array}$ & $\begin{array}{c}91.40 \\
\%\end{array}$ & $\begin{array}{c}299,773,880 \\
.20 / \\
328,567,670 \\
.41\end{array}$ & $91 \%$ \\
\hline \multirow{2}{*}{$\begin{array}{c}\text { Disbursem } \\
\text { ents as \% } \\
\text { of } \\
\text { Obligation } \\
\text { s }\end{array}$} & $\begin{array}{l}2018 \\
(d / c)\end{array}$ & $\begin{array}{c}102,414,046 \\
.83 / \\
102,426,274 \\
.18\end{array}$ & $\begin{array}{c}99.99 \\
\%\end{array}$ & $\begin{array}{c}146,814,158 \\
.35 / \\
150,923,865 \\
.78\end{array}$ & $97.28 \%$ & $\begin{array}{c}75,257,591 . \\
14 / \\
75,239,569 . \\
49\end{array}$ & $\begin{array}{c}100.02 \\
\%\end{array}$ & $\begin{array}{c}324,485,796 \\
.32 / \\
328,589,709 \\
.45\end{array}$ & $99 \%$ \\
\hline & $\begin{array}{l}2017 \\
(h / g)\end{array}$ & $\begin{array}{c}91,835,687 . \\
86 / \\
91,847,254 .\end{array}$ & $\begin{array}{c}99.99 \\
\%\end{array}$ & $\begin{array}{c}132,861,415 \\
.86 / \\
140,580,063\end{array}$ & $94.51 \%$ & $\begin{array}{c}42,782,203 . \\
93 / \\
46,114,144 .\end{array}$ & $\begin{array}{c}92.77 \\
\%\end{array}$ & $\begin{array}{c}267,479,307 \\
.65 / \\
278,541,462\end{array}$ & $96 \%$ \\
\hline
\end{tabular}




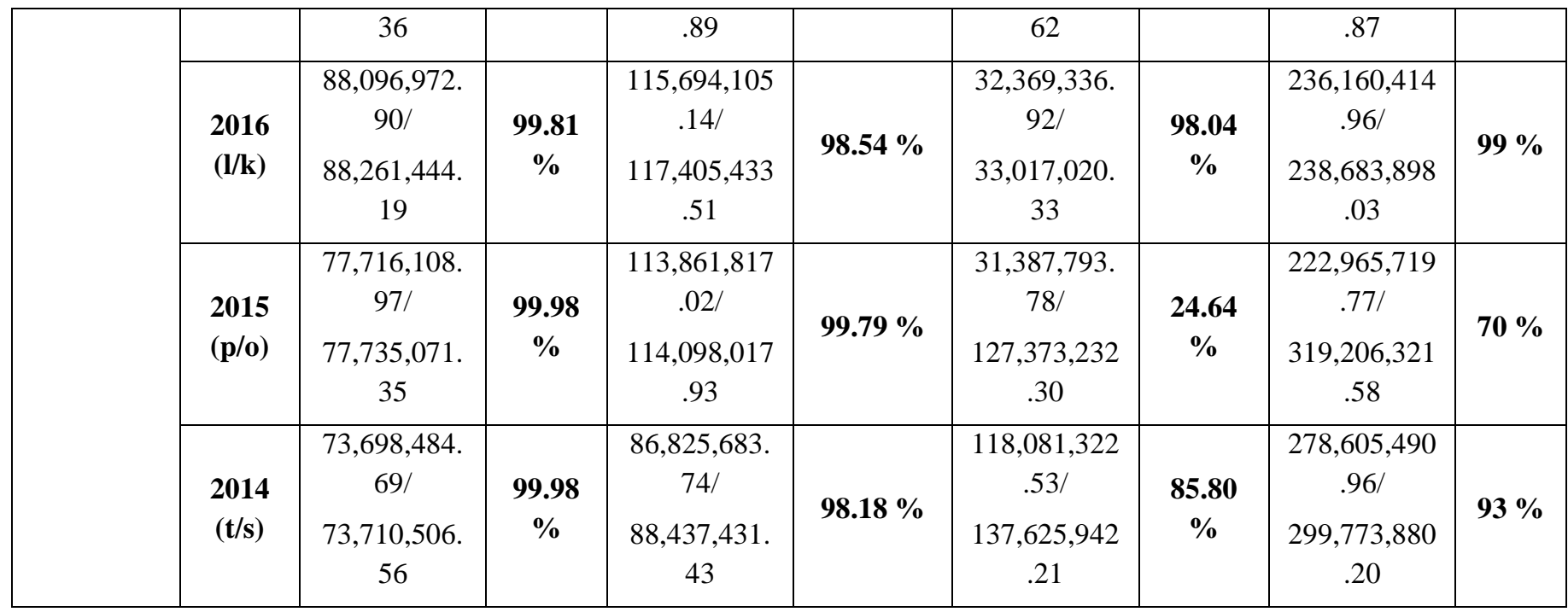

Source/s: Statement of Appropriations, Allotments, Obligations, and Balances (SAAOB) and SREs of covering fiscal years.

Table 4 reveals that in all the last five years, total released allotments for each allotment class were $90-100 \%$ of the total appropriation, then total actual obligations were above 90\% from 2014-2017 of the total allotments released, lastly, for four years total actual disbursements were more than $90 \%$ of the total obligations. The fall below $90 \%$ of expenditures and disbursements in years 2018 and 2015 occurred to secure net debt service ceiling and borrowing capacity by the year-end financial reports for loans and borrowings of the LGU. Still, more than $90 \%$ determines that the amount duly appropriated was spent as intended for approved appropriation ordinances, and the magnitude of obligations paid by the LGU.

Table 5. Indicator \# 9 - Real Property Tax (RPT) Accomplishment Report

\begin{tabular}{|l|c|c|c|c|c|}
\hline \multicolumn{1}{|c|}{ Real Property Taxes Revenues } & $\mathbf{2 0 1 8}$ & $\mathbf{2 0 1 7}$ & $\mathbf{2 0 1 6}$ & $\mathbf{2 0 1 5}$ & $\mathbf{2 0 1 4}$ \\
\hline ESTIMATED & $22,324,000.00$ & $19,310,000.00$ & $17,103,300.00$ & $14,985,000.00$ & $12,951,317.00$ \\
\hline ACTUAL & $15,467,480.15$ & $11,345,072.40$ & $10,085,956.75$ & $9,056,907.60$ & $7,908,751.00$ \\
\hline $\begin{array}{l}\text { Actual Revenues as \% of Estimated } \\
\text { Revenues }\end{array}$ & $\mathbf{6 9 \%}$ & $\mathbf{5 9 \%}$ & $\mathbf{5 9 \%}$ & $\mathbf{6 0} \%$ & $\mathbf{6 1 \%}$ \\
\hline
\end{tabular}

Source/s: Municipal Assessor's Reports on Taxable Real Property Tax limited to LGUs share and SREs

It reveals that in all the last five years, Real Property Tax Accomplishment was less than 70\%. It is a signal that LGU has not fully exercised its potent resources. Regular updates of the Revenue Code and schedule of market values are needed to improve the collection efficiency rate of the LGU.

Table 6. Indicator \# 10 - Effectiveness of Tax Enhancement Measures (Delinquent Real Property Tax)

\begin{tabular}{|c|c|c|c|c|c|c|}
\hline \multicolumn{6}{|c|}{ Real Property Tax (RPT) Delinquencies } & \multirow{2}{*}{$\begin{array}{l}\% \text { of Increase } \\
\text { in Collection }\end{array}$} \\
\hline Amount Due & $\begin{array}{c}\text { Actual } \\
\text { Collections }\end{array}$ & $\%$ Collected & Amount Due & $\begin{array}{c}\text { Actual } \\
\text { Collections }\end{array}$ & $\begin{array}{c}\% \\
\text { Collected }\end{array}$ & \\
\hline \multicolumn{3}{|c|}{ Next Preceding Year's Collection (2017) } & \multicolumn{3}{|c|}{ Immediately Preceding Year's Collection (2018) } & \multirow{2}{*}{$-11 \%$} \\
\hline $32,833,217.14$ & $15,839,101.46$ & $48 \%$ & $46,039,393.40$ & $16,944,912.80$ & $37 \%$ & \\
\hline \multicolumn{3}{|c|}{ Next Preceding Year's Collection (2015) } & \multicolumn{3}{|c|}{ Immediately Preceding Year's Collection (2016) } & \multirow{2}{*}{$21 \%$} \\
\hline $46,884,938.68$ & $12,892,958.26$ & $27 \%$ & $28,236,598.46$ & $13,516,397.80$ & $48 \%$ & \\
\hline
\end{tabular}

Source/s: LGUs Real Property Tax Account Register-Certified List of All RPT delinquent accounts settled for the next preceding and immediately preceding years of covering fiscal years. 
Table 6 shows that year 2015-2016, RPT collection strategies contributed to at most a $20 \%$ increase in the percentage of delinquent RPT collected in the last two years while in the year 2017-2018, RPT collection strategies did not contribute to an increase for last two years. Since, there is difficulty in the participation of different barangays in the tax information drive of the Municipality, and coordination of the Provincial Treasurer in the application of administrative remedies for delinquent taxpayers needed to improve voluntary compliance of taxpayers to minimize delinquent accounts. Hence, the tax efficiency rate is relatively low and tax enhancement measures became ineffective.

Table 7. Indicator \# 11 - Predictability in the availability of cash for commitment of expenditures

\begin{tabular}{|c|c|r|r|r|r|c|}
\hline Year & Current Year & $\begin{array}{c}\text { Continuing } \\
\text { Appropriations }\end{array}$ & $\begin{array}{c}\text { Prior } \\
\text { Year's } \\
\text { Liabilities }\end{array}$ & Total & Cash Available & $\begin{array}{c}\text { \% of Allotment with } \\
\text { Available Cash }\end{array}$ \\
\hline $\mathbf{2 0 1 8}$ & $568,626,509.75$ & $15,700,530.17$ & $417,992.85$ & $584,745,032.77$ & $584,745,032.77$ & $\mathbf{1 0 0 \%}$ \\
\hline $\mathbf{2 0 1 7}$ & $307,289,483.04$ & $7,402,841.55$ & $370,686.40$ & $315,063,010.99$ & $316,877,222.39$ & $\mathbf{1 0 1 \%}$ \\
\hline $\mathbf{2 0 1 6}$ & $263,311,824.04$ & $100,182,651.73$ & $102,100.00$ & $363,596,575.77$ & $375,054,266.68$ & $\mathbf{1 0 3 \%}$ \\
\hline $\mathbf{2 0 1 5}$ & $243,309,706.83$ & $1,737,014.27$ & $279,634.33$ & $245,326,355.43$ & $249,001,431.79$ & $\mathbf{1 0 1 \%}$ \\
\hline $\mathbf{2 0 1 4}$ & & $572,390.45$ & $27,456.01$ & $329,167,516.87$ & $333,876,602.18$ & $\mathbf{1 0 1 \%}$ \\
\hline
\end{tabular}

Source/s: LGUs Last five years' SAOBs and Statement of Cashflows

Table 7 displays that in all the last five years, 100\% of allotments to support budgeted programs, projects, activities (PPAs) including liabilities have available cash. As the LGU exercised cash program-flow analysis, financial and physical performance targets as financial control tools in controlling cash outflows matched with cash inflows ensure that sufficient cash is available to settle obligations as they fall due.

Table 8. Indicator \# 12 - Value for Money and Controls in Procurement

\begin{tabular}{|l|r|r|}
\hline \multicolumn{1}{|c|}{$\begin{array}{c}\text { LGUs Annual Procurement Plan- Common-Use Supplies and Equipment (CSE) } \\
\text { Monitoring Report and Agency Purchase Requests (APRs) }\end{array}$} & $\begin{array}{r}\text { Fiscal Years } \\
\mathbf{2 0 1 8 - 2 0 1 6}\end{array}$ & $\begin{array}{c}\text { Fiscal Years } \\
\mathbf{2 0 1 6}-2014\end{array}$ \\
\hline Total Amount of CSE Purchased for the Year (a) & $\mathbf{1 4 , 3 3 0 , 6 1 0 . 3 7}$ & $\mathbf{9 , 6 9 0 , 2 8 0 . 4 0}$ \\
\hline Amount of CSE Covered by APRs Submitted to DBM-PS (b) & $\begin{array}{r}\mathbf{P} \\
\mathbf{0 . 0 0}\end{array}$ \\
\hline $\begin{array}{l}\text { \% of Total Amount of CSE Purchased covered by APRs submitted to DBM-PS (c }= \\
\text { b/a) }\end{array}$ & $\mathbf{2 , 4 8 6 , 3 6 3 . 6 5}$ \\
\hline Amount of CSE Actually Purchased from DBM-PS (d) & $\mathbf{1 7 . 3 5 \%}$ \\
\hline $\begin{array}{l}\text { Total no. of procurement activities undertaken as per approved Annual/Supplemental } \\
\text { Plan (A/S PP) }\end{array}$ & $\mathbf{4 4 1 , 0 3 6 . 9 2}$ \\
\hline \% of Actual procurement in accordance with the approved A/S PP (c = a / b) & $\mathbf{0 . 0 0}$ \\
\hline
\end{tabular}

Source/s: LGUs Approved Annual/Supplemental Procurement Plan-Common Use Supplies and Equipment (APP-CSE), Procurement Monitoring Reports, and Agency Purchase Requests (APRs).

Table 8 states that only $17.35 \%$ of purchases of common-use supplies and equipment were covered by APRs submitted to DBM-PS and Annual/Supplemental Procurement Plan approved by $100 \%$ of procurement activities. Many supplies have been acquired using alternate law-sanctioned processes.In compliance with R.A.No 9184 and its IRR, nearly all procurement operations were not posted within the specified time in the PhilGEPS. Whilst $100 \%$ of requested goods and services are delivered/completed on time.

This article can be downloaded from here: www.ijaems.com 
Table 9. Indicator 13 \& 14. Effectiveness of Payment Controls for PS and Non-PS Expenditures

\begin{tabular}{|l|r|r|}
\hline \multicolumn{1}{|c|}{ Allotment Class } & Fiscal Year (2018) & $\begin{array}{c}\text { Fiscal Year } \\
(\mathbf{2 0 1 6})\end{array}$ \\
\hline Total Personal Services (PS) Expenditures for the immediately preceding year (a) & P102,414,046.83 & P $88,096,972.90$ \\
\hline Total PS Expenditures which have adverse COA findings (b) & - \\
\hline Total PS Expenditures which have no adverse COA findings (c = a-b) & $\mathbf{P 1 0 2 , 4 1 4 , 0 4 6 . 8 3}$ & $\mathbf{P ~ 8 8 , 0 9 6 , 9 7 2 . 9 0}$ \\
\hline \% of PS Expenditures which has no adverse COA findings (d = c/a) & $\mathbf{1 0 0} \%$ & $\mathbf{1 0 0} \%$ \\
\hline Total Non-PS Expenditures for the immediately preceding year (a) & $\mathbf{2 6 4 , 5 0 9 , 1 9 2 . 8 4}$ & P 236,145,311.35 \\
\hline Total Non-PS Expenditures Disallowed in Audit (b) & $\mathbf{2 6 4 , 5 0 9 , 1 9 2 . 8 4}$ & $\mathbf{P ~ 2 3 6 , 1 4 4 , 8 3 1 . 3 5}$ \\
\hline Total Non-PS Expenditures Allowed in Audit (c = a-b) & $\mathbf{1 0 0} \%$ & $\mathbf{1 0 0 \%}$ \\
\hline \% of Non-PS Expenditures Allowed in Audit (d = c/a) & \\
\hline
\end{tabular}

Source/s: LGUs immediately preceding year's SAOBs, and Annual Audit Report (AAR)

Table 9 indicates that $100 \%$ there are no adverse COA audit findings for Personal Services (PS) and Non-PS (MOOE \& Capital Outlays) expenditures. The results of "no adverse COA findings" imply adherence of the LGU in personal services limitations for the expenditure program. Reconciliation/liquidations of accounts, the quality, and timeliness of regular financial reports of the LGU are made consistently.

\section{CONCLUSIONS AND RECOMMENDATIONS}

The study showed the strength of LGU-Talavera lies in its sound accounting, recording, and reporting of financial statements and overall participation of Citizens in the planning and budgeting process. Although internal and external audit dramatically increases, it contributes to the pitfalls of LGU if not taken actions along with the predictability of budget execution. On the financial performance, total actual Local Revenue collections compared to estimated are yearly improving. However, the real property tax accomplishment rate was not realized and RPT enhancement measures for delinquent accounts are not effectively implemented. Though requested goods and services, civil works, and consulting services are delivered/completed on time, the procurement process is partially complied with by the LGU. Financial requirements for operations of all local economic enterprises and public utilities were funded and allotments to support budgeted programs, projects, activities (PPAs) including liabilities have available cash. Thus, obligations are meet effectively and assert to financial soundness.

In 2014-2016 \& 2016-2018, a considerable improvement was achieved in the evaluated PFM System of LGU-Talavera, N.E. The average overall description rate indicates that the elements are open and organized however, not entirely functional. Furthermore, the LGU needs to improve performance $\{[28]$, [29], [30]\} on each critical dimension by formulating measures on the elements of the PFM system to be effective.PFMAT, especially in LGU-Talavera, is an effective LGU performance measurement method. It is comprehensive in its performance indicators of each critical dimension. It can accurately track the successes and limitations and offers a straightforward analysis of a municipality's overall financial results, helping to prepare and execute improvements for the LGU itself. It can compare previous and current success accurately and plan where to reflect on maintaining good financial potential in the future. While PFMAT has successfully chronicled the performance and development of LGUs PFM, it still recognizes that the use of public finances for PFMAT continues to face numerous challenges to satisfy its current utility. To retain its effectiveness in enhancing the financial capabilities of LGUs, PFMAT should, therefore, be continuously improved and adapted to the ever-changing concept of the public financial management system and evaluation.

The study showed and identifiedthe need [31] to further understand the full potential of local government units in updating and implementation of local revenue codes especially the Real property tax which is the biggest and most potent source of revenues. Local government units should establish a more comprehensive study in the Real Property Tax enhancement measures to exert more efforts in the collection of RPT. Request for Condonation of Penalties and Interest to minimize the delinquent accounts to further strengthen the revenues needed for the implementation of long-term plans under the Capacity Development Program of the LGU. More collection efforts 
as a means of lessening the municipality's dependence on the Internal Revenue Allotment (IRA) and realize its goal for accelerated economic growth. Suggestions on the full compliance of the municipality regarding the activities in the procurement process following the RA 9184 and its IRR and the follow-up process on the audit to foster transparency, effectiveness, and efficiently obtain the best value for money on all government operations. It is also recommended that a regular and timely rating may be conducted for each LGU and posted to the DBM website promptly to provide real-time data for researchers. Public Financial Management systems are crucial in the establishment of Good Local Governance. A comparative study on the public financial management of highperforming LGUs may be conducted as a follow-up study to benchmark the best practices to be adopted by lowperforming LGUs.

\section{REFERENCES}

[1] Swirska, Anna (2016). Methods for Improving the Effectiveness of the Financial Management of Local Government Units in Poland

[2] De Renzio, P. (2015). PFM systems performance measures. GSDRC Prof-Dev't Reading File no. 28. Birmingham, UK: University of Birmingham

[3] Department of Budget and Management (2012). Public Financial Management Assessment Tool Book for Local Government Units (PFMAT Book for LGUs) p.1, p.3, p.4, p.2,

[4] ACCA, (2010). Improving public sectors financial management in emerging economies and developing countries. Chartered Certified Accountants Association

[5] Parry, M. (2010). The Public Financial Management - Four Dimensions

[6] Cangiano, M., et.al @ 2013 IMF- Emerging Architecture for Public Financial Management p.3

[7] IMF (2014). Fiscal transparency initiative update. IMF Policy Paper. Washington, DC: International Monetary Fund

[8] CABRI (2009). Budget Practices and Procedures in Africa. Pretoria: Collaborative Africa Budget Reform Initiative.

[9] World Bank, PEFA Secretariat (2005). Measurement Framework for Public Financial Management Performance.

[10] PEFA Secretariat (2016). PFM Framework for Assessment. www.pefa.org

[11] World Bank (2016). Update version of PEFAs framework for assessment of PFM performance

[12] OECD (2017), OECD Budget Transparency Toolkit: Practical Steps for Supporting Openness, Integrity \& Accountability in Public Financial Management.

[13] Mackie, A. (2011). PFM Diagnostic Instruments Stocktaking Study p. 15

[14] Ndunguru, Nicholaus S. (2013).The Effect of Financial Management Systems on Local Government Performance: The Case of Namtumbo District Council
[15] Odd - Helgefjelstsd, et al. (2006). Local Government Finances and Financial Management in Tanzania. Special Paper No. 16 Observation from Six Councils

[16] DFAT, (2010). Philippines-Australia Programs on Public Financial Management

[17] World Bank, PEFA Secretariat (2019). Contribution of PEFA to the Philippines PFM Reform Roadmap, https://www.pefa.org/news/pefas -contribution-philippinespfm-reform-roadmap

[18] World Bank Philippines Development Trust Fund, ADB and IMF (2016) Philippines' PFM Strategy Implementation Support: PFM and Accountability Assessment

[19] DBM (2012). PFMAT Book for LGUs p.3

[20] DBM (2012). PFMAT Book for LGUs p.4

[21] DBM (2012). PFMAT Book for LGUs p.2

[22] Gay (1992), Descriptive Research Method Retrieved from http://repo.iain-

tulungagung.ac.id/4550/4/CHAPTER\%20III.pdf

[23] Municipality Profile of Talavera, Province of Nueva Ecija (2015). Retrieved from https://www. talavera .gov.ph/abouttalavera/municipal-profile/

[24] DBM (2012). PFMAT Book for LGUs p.19

[25] DBM (2012). PFMAT Book for LGUs p.15

[26] Mcdermott, Haley, (2013). Quigley, Martin Logic Model. Retrieved from: https://planningforconflict.wordpress.com/2013/10/09/logicmodel/

[27] DBM (2012). PFMAT Book for LGUs p.36

[28] Subia, G., Amaranto, J., Amaranto, J., Bustamante, J., \& Damaso, I.(2019) Chess and Mathematics Performance of College Players: An Exploratory Analysis. Open Access Library Journal, 6, 1-7.doi: 10.4236/oalib.1105195.

[29] Pentang,J. T.,Ibañez, E. D., Subia, G. S., Domingo, J. G., Gamit, A. M., \& Pascual, L. E.(2021). Problem-solving performance and skills of elementary prospective teachers in Northern Philippines. Journal of Hunan University Natural Sciences, 48(1), 122-132.

[30] Marcos, M., et.al. (2020). Entry Competencies andPerformance in Mathematics of First-Year Engineering Students in State Universities in Region 3. Test Engineering and Management. ISSN: 0193-4120 Page No. 10148 10158.

[31] Mina, J.C., Barlis, P.T., Vega, N.C. and Subia, G.S. (2019) Corporate Social Responsibilities of Selected Resorts in Cabanatuan City, Nueva Ecija, Philippines. Open Access Library Journal, 6: https://doi.org/10.4236/oalib.1105292 